\title{
Radiological Analysis of Symptomatic Complications after Bilateral Laminotomy for Lumbar Spinal Stenosis
}

\author{
Jong Hun Seo ${ }^{1}$, Gun Park ${ }^{2}$, Chang $\|_{\text {Ju}}{ }^{1}$, Seok Won Kim', Seung Myung Lee ${ }^{1}$ \\ Departments of Neurosurgery ${ }^{I}$ and Laboratory, Chosun University College of Medicine, Gwangju, Korea
}

\begin{abstract}
Objective: The purpose of this study was to describe the various symptomatic complications following decompressive bilateral laminotomy for treating lumbar spinal stenosis.

Methods: Out of 878 patients who underwent decompressive laminotomy from 2006 through 2008, 178 patients who were able to be followed for a minimum of 24 months were included in this study. The mean age at the time of surgery was 64.4 years (range, 38-79), and the average follow-up period was 32.7 months. The development of symptomatic complications was observed using simple radiographs and MR imaging during the follow-up period. Imaging and clinical compli- cations were focused on postoperative spondylolisthesis, disc herniation, and facet cysts, and were analyzed according to developmenttime, the degree of symptom, and their course.

Results: Postoperative simple radiographs revealed that eight out of 178 patients $(4.4 \%)$ had developed symptomatic spondylolisthesis and six required an interbody fusion procedure. Five patients with disc herniation (2.8\%) at the level of the decompressive bilateral laminotomy site underwent another operation within 24 months after the first surgery. In two patients, disc herniations developed within 1 month after surgery. Three (1.7\%) out of 178 patients showed facet cysts but all symptoms were relieved by conservative treatment. Spontaneous regression of the cysts was observed during the follow-up period. Conclusions: Although decompressive bilateral laminotomy for spinal stenosis is believed to effective and reduces the need for fusion, various symptomatic complications were observed after this procedure. It is important to be aware of the possibility for these various complications to improve the surgical outcome.
\end{abstract}

Key Words: Stenosis $\cdot$ Laminotomy $\cdot$ Complication

\section{INTRODUCTION}

Lumbar spinal stenosis (LSS) is being increasingly diagnosed in the elderly by advanced imaging studies. For symptomatic patients, the goal of surgery is to relieve pain and to improve spinal function while preserving the normal anatomy. The primary goal of the procedure is to achieve adequate decompression of the neural elements ${ }^{7}$. Minimally invasive procedures have been increasingly used to treat LSS. With the development of minimally invasive surgical techniques, more limited decompressive procedures including simple ligamentectomy following bilateral laminotomy or unilateral laminectomy for bilateral decompression have been shown to be ef-

- Received: February 2, 2012 - Revised: March 26, 2012

- Accepted: March 28, 2012

Corresponding Author: Seok Won Kim, MD

Department of Neurosurgery, Chosun University College of Medicine,

588, Seosuk-dong, Dong-gu, Gwangju-city 501-717, Korea

Tel: +82-62-220-3126, Fax: +82-62-227-4575

E-mail: ns64902@hanmail.net fective and are known to reduce the need for fusion ${ }^{5,12)}$. However, even minimal invasive procedures are not free of complications. Procedure-related complications include postoperative spondylolisthesis, lumbar disc herniation, and development of facet cysts. The purpose of this study was to analyze the complications of decompressive bilateral laminotomy focusing on factors that influenced poor surgical results.

\section{MATERIAL AND METHODS}

Out of 878 patients who underwent decompressive bilateral laminotomy without fusion for LSS from January 2006 to December 2008, data from 178 patients who were able to be followed for a minimum of 24 months were reviewed. The patient population consisted of 82 men and 96 women whose mean age at the time of surgery was 64.4 years (range, 38-79 years). The demographic and preoperative data of the patients are shown in Table 1. Major complications such as surgical site infection, dural tears, and nerve injury were not investigated. The follow-up period after the surgery ranged 
from 24 to 49 months (mean, 32.7 months). All patients met the following inclusion criteria: (1) narrowing of the central portion of the spinal canal with an area of the dural sac measuring less than $80 \mathrm{~mm} 2$ at the intervertebral level, and (2) neurogenic claudication as defined by leg pain that limited standing, ambulation, or both. The exclusion criteria were: (1) previous back surgery, (2) decompression for malignancy or infection, (3) decompression with instrumented fusion, and (4) the presence of preoperative instability, spondylolisthesis, or combined disc herniation. All patients underwent bilateral laminotomy and simple ligamentectomy for lumbar spinal stenosis (Fig. 1). We tried to avoid performing medial facetectomy to maintain spinal stability.

\section{Clinical and radiologic evaluation}

The development of complications was determined by simple radiographs and MR imaging during follow-up. In addition, MR imaging was performed when the symptoms appeared or worsened during the follow-up period after surgery. Furthermore, wecompared the demographic data and radiographic findings of patients without postoperative complications to evaluatethe factors possibly associated with the causes

Table 1. Demographic and preoperative data

\begin{tabular}{ll}
\hline \hline Variable & \multicolumn{1}{c}{ Value } \\
\hline Mean age & 64.4 (38-79) \\
Sex (M:F) & $82: 96$ \\
Type of neurogenic claudication & \\
$\quad$ cauda equine type & 137 \\
$\quad$ radiculopathy & 41 \\
Surgical levels & \\
L2-L3 & 1 \\
L3-L4 & 9 \\
L4-L5 & 90 \\
L5-S1 & 15 \\
L3-L4, L4-L5 & 47 \\
L4-L5, L5-S1 & 12 \\
L2-L3, L3-L4, L4-L5 & 4 \\
Mean follow-up period (months) & $32.4(24-49)$ \\
\hline
\end{tabular}

of symptomatic complications. As mentioned above, this study included only clinical complications assessed by radiological methods, so other major complications such as neural injury, dural tearing, and deep wound infection were not investigated in this study.

\section{RESULTS}

Seventeen different symptomatic complications (8.9\%) were observed during the follow-up period in the present study. Therewere three types of clinical complications assessed by radiological studies after decompressive bilateral laminotomy

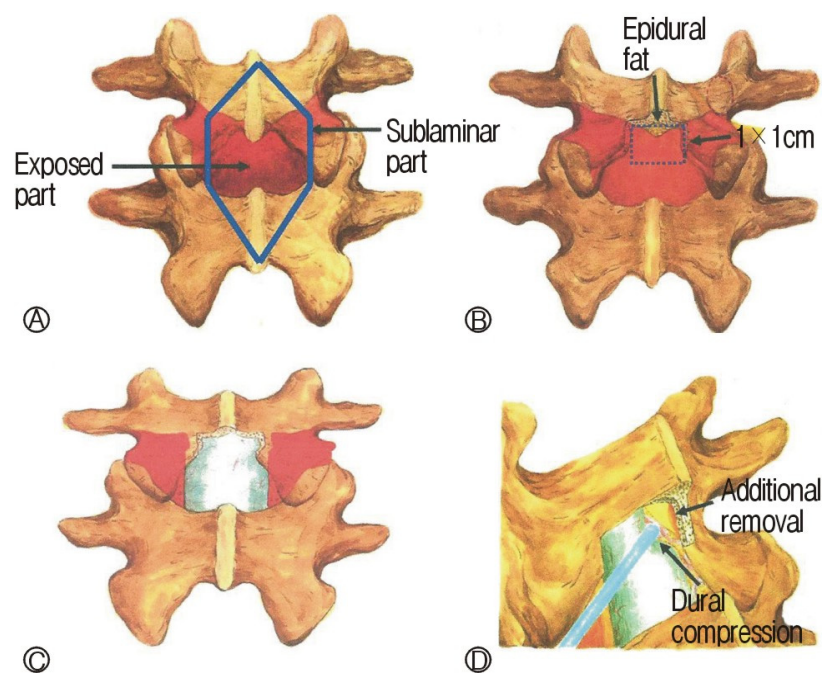

Fig. 1. Surgical Procedure of bilateral laminotomy and ligamentectomy. A: Midline was incised on the supraspinous and infraspinous ligaments and spinous processes and lamina were elevated subperiostealy and paraspinal muscles were retracted bilaterally. B: Removal of lower 1/3 of upper spinous process to the base and then expose yellow ligament underneath C: Detachment of sublaminar part with curved sharp-tipped freer and removal of exposed part of the yellow ligament. D: After tilting of patient to opposite side from the operator the lateral sublaminar part of ligament was detached and removed and mesial facetectomy was performed if needed.

Table 2. Summary of patients with postoperative spondylolisthesis

\begin{tabular}{cllcll}
\hline \hline Case & Age/Sex & Surgical levels & Level of & spondyloisthesis Duration until spondylolisthesis & Course \\
\hline 1 & $68 / F$ & L4-L5 & L4-L5 & 27 months & PLIF \\
2 & $72 / F$ & L4-L5 & L4-L5 & 12 months & PLIF \\
3 & $70 / F$ & L4-L5 & L4-L5 & 12 months & Conservative treatment \\
4 & $46 / M$ & L3-L4, L4-L5 & L4-L5 & 27 months & PLIF \\
5 & $59 / M$ & L4-L5 & L4-L5 & 24 months & PLIF \\
6 & $60 / F$ & L3-L4, L4-L5 & L4-L5 & 12 months & Conservative treatment \\
7 & $66 / M$ & L3-L4, L4-L5 & L3-L4 & 16 months & PLIF \\
8 & $69 / F$ & L4-L5 & L4-L5 & 17 months & PLIF \\
\hline
\end{tabular}


for lumbar spinal stenosis.

\section{Spondylolisthesis}

Spondylolisthesis developing after surgery with a displacement of more than $5 \mathrm{~mm}$ was observed in eight patients. The average degree of displacement was $6.5 \mathrm{~mm}$ after surgery. All postoperative spondylolisthesis developed at least 12 months after surgery at the L4-L5 levels except one case. The symptoms associated with the development of spondylolisthesis were persistent or new low back pain and radiculopathy. Six out of eight patients required an interbody fusion procedure (Fig. 2).
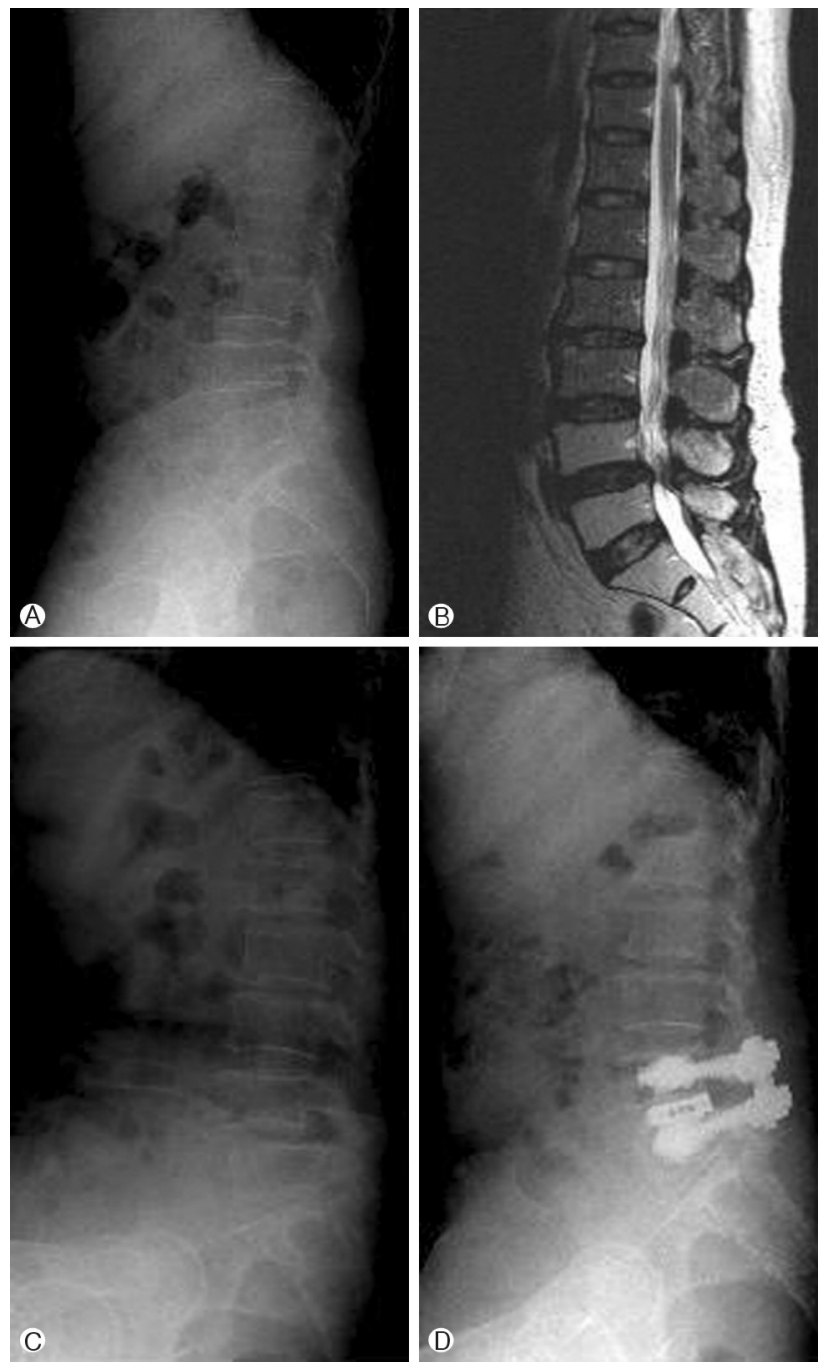

Fig. 2. A 68-year-old woman with L4-L5 spinal stenosis. A \& B: Simple lateral radiograph and T2-weighted magnetic resonance image reveal spinal stenosis at L4-L5 without spondylolisthesis. C: Progression of spondylolisthesis is observed at 27 months after surgery. D: Simple lateral radiograph shows posterior lumbar interbody fusion status.

\section{Disc herniation in the operated segments}

The main reasons for poor outcomes in five patients seemed to be due to new disc herniation in the resected segments that caused persistent or sudden low back and leg pain. Although four of these patients underwent microscopicdiscectomy without fusion and one patient underwent fusion as a second surgery, their final outcomes were not satisfactory mainly because of low back pain. All new lumbar disc herniation occurred at the L4L5 level (Table 3). Moreover, two of these cases occurred within 1 month after decompressive bilateral laminotomy (Fig. 3).

\section{Facet cysts}

Postoperative intraspinal facet cysts developed in three patients (1.7\%) during the follow-up period. The cysts developed at the L4-L5 level in all patients. All cysts developed within 12 months after surgery and facet fluid was observed on preoperative MR imaging. The exhibited symptoms were bilateral
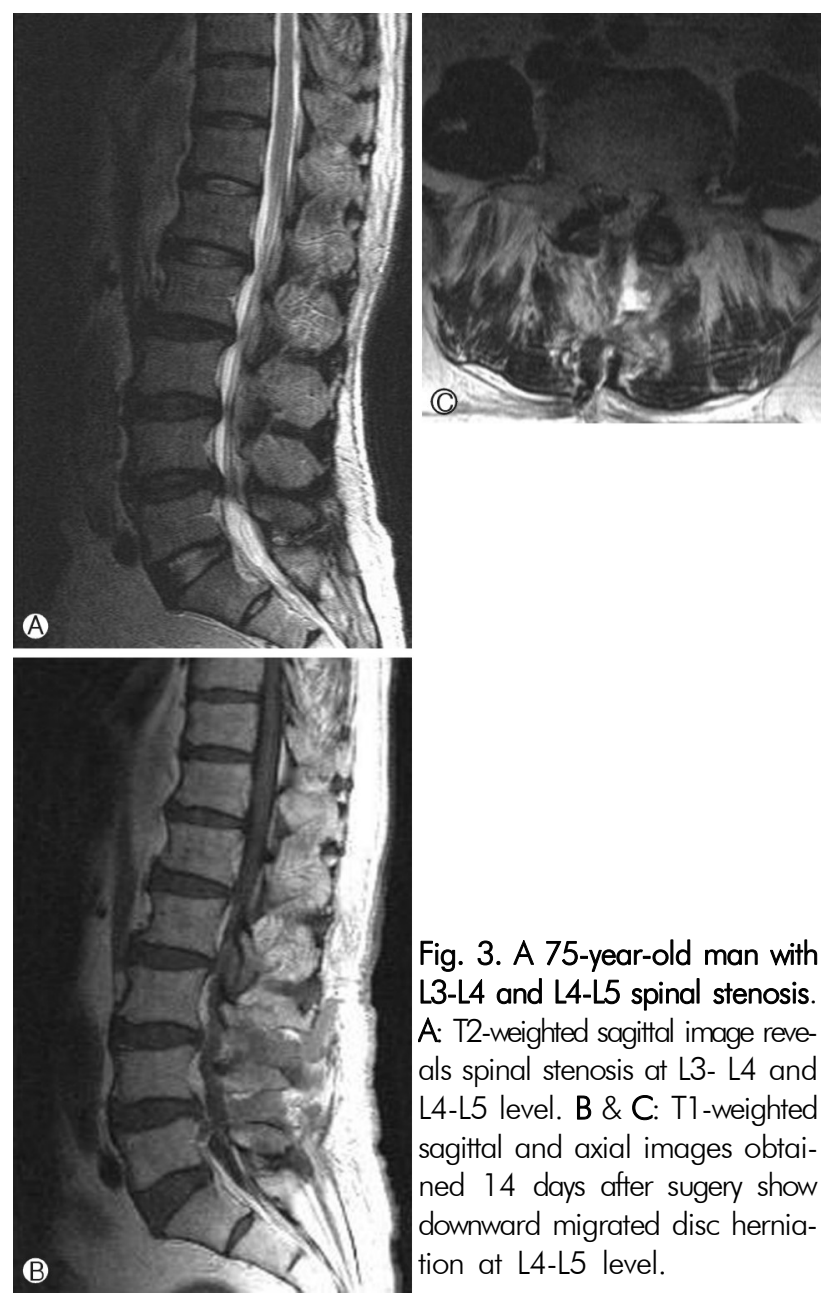

Fig. 3. A 75-year-old man with L3-L4 and L4-L5 spinal stenosis. A: T2-weighted sagittal image reveals spinal stenosis at L3- $\mathrm{L} 4$ and L4-L5 level. B \& C: T1-weighted sagittal and axial images obtained 14 days after sugery show downward migrated disc herniation at L4-L5 level. 
and they were relieved by conservative treatment. Spontaneous regression of the cysts was observed in all patients (Fig. 4).

\section{DISCUSSION}

An aging population and changing life styles have increased the incidence of LSS. This disorder is the most common surgical indication for geriatric patients. Traditional total laminectomy with or without fusion remains the standard surgical treatment for LSS. However, traditional bilateral laminectomy is associated with numerous postoperative complications, including vertebral instability, severe atrophy of paraspinal muscles, and posterior scarring, which can leadto poor surgical results, ${ }^{5,11}$. A variety of less extensive procedures have been proposed in an attempt to preserve normal spinal anatomy. In this study, the surgical procedure carried out was bilateral laminotomy following removal of the posterior third of the spinous process. This procedure achieved complete decompression of the nerve root through removal of the ligamentum

Table 3. Summary of patients with disc herniation at surgical level

\begin{tabular}{cllclcl}
\hline \hline Case & $\begin{array}{c}\text { Age/ } \\
\text { Sex }\end{array}$ & Surgical levels & $\begin{array}{c}\text { Level of disc } \\
\text { herniation }\end{array}$ & \multicolumn{1}{c}{ Symptoms } & $\begin{array}{c}\text { Duration until } \\
\text { disc herniation }\end{array}$ & Course \\
\hline 1 & $75 / M$ & L3-L4, L4-L5 & L4-L5 & Back pain\& radioculopathy & 14 days & Endoscopic discectomy \\
2 & $62 / F$ & L4-L5 & L4-L5 & Back pain\& radioculopathy & 18 months & Microscpic discectomy \\
3 & $49 / M$ & L4-L5 & L4-L5 & radioculopathy & 3 months & Microscpic discectomy \\
4 & $68 / F$ & L3-L4, L4-L5 & L4-L5 & Back pain\& radioculopathy & 12 months & Microscpic discectomy \\
5 & $65 / M$ & L4-L5 & L4-L5 & Back pain\& radioculopathy & 21 days & PLIF \\
\hline
\end{tabular}

Table 4. Summary of patients with postoperative intraspinal facet cysts

\begin{tabular}{cccccccc}
\hline \hline Case & $\begin{array}{c}\text { Age/ } \\
\text { Sex }\end{array}$ & $\begin{array}{c}\text { Surgical } \\
\text { levels }\end{array}$ & $\begin{array}{c}\text { Level of } \\
\text { cysts }\end{array}$ & Laterality & $\begin{array}{c}\text { Duration until cyst } \\
\text { development (months) }\end{array}$ & Symptoms & $\begin{array}{c}\text { Course of cysts on Facet fluid } \\
\text { MRI/months } \\
\text { on MRI }\end{array}$ \\
\hline 1 & $64 / M$ & L3-L4, L4-L5 & L4-L5 & Bilateral & 9 & Back pain \& radiculopathy & regression/24 \\
2 & $63 / F$ & L4-L5 & L4-L5 & Bilateral & 6 & Radiculopathy & regression/10 \\
3 & $71 / M$ & L3-L4, L4-L5 & L4-L5 & Bilateral & 3 & Back pain \& radiculopathy & regression/36 \\
+
\end{tabular}
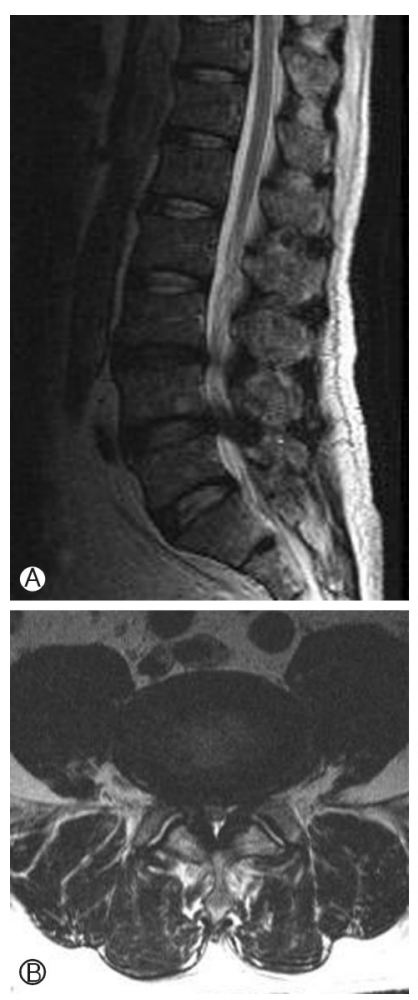
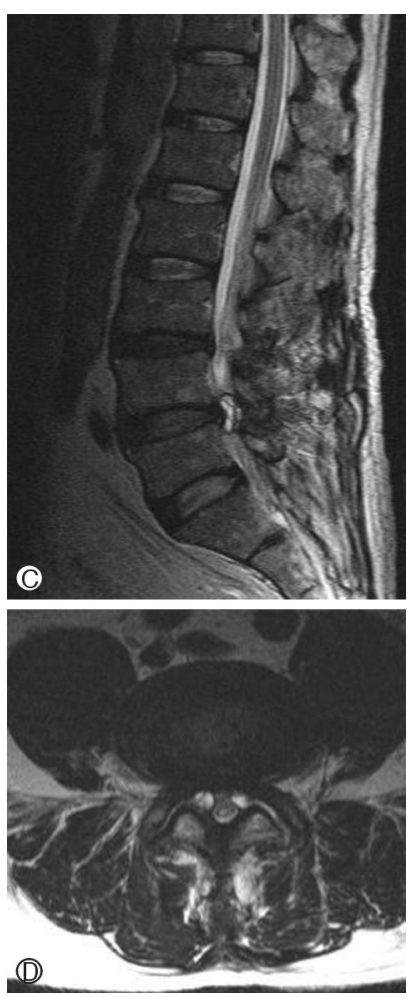
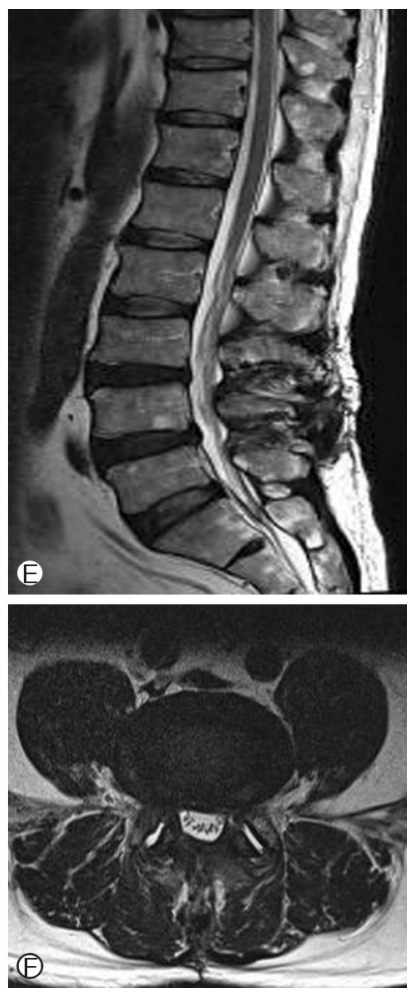

Fig. 4. A 64-year-old man with spinal stenosis at L3-L4 and L4L5 level. A \& B: Preoperative magnetic resonance images show spinal stenosis at L3-L4 and L4-L5 level. C \& D: Magnetic resonance images obtained 9 months after surgery reveal bilateral intraspinal facet cysts. $E \& F$ : the regression of the cysts were documented on magnetic resonance images obtained 2 years after surgery. 
flavum occupying the lateral recess while minimizing damage to facet joints. This procedure allows more effective decompression while minimizing nerve root irritation. Decompressive bilateral laminotomy and flavectomy without spinal fusion has been the first choice of surgical treatment for degenerative spinal stenosis at our institu- tion for patients with no instability or spondylolisthesis. We believe it has become a standardtreatment for LSS for improving the quality of life. Despite the simplicity and safety of this procedure compared to traditional wide laminectomy with or without fusion, several complications may develop. In our study, we encountered primarilythree kinds of complications after bilateral laminotomy and flavectomy.

\section{Spondylolisthesis}

Spondylolisthesis or spinal instability is a relatively wellknown potential long-term complication of stand-alone decompressive laminectomy procedures" ${ }^{9}$. It is believed that sparing the posterior elements, not only the laminae and the functionally important parts of the facets but also the interspinous ligament, can be beneficial for preventing spinal instability. Although we performed fenestration with minimal soft tissue dissection and limited bone removal instead of extensive laminectomy, we conclude that bilateral laminotomy and ligamentectomy can also cause postoperative slippage in patients without preoperative spondylolisthesis. The resection of stability-preserving structures may promote operation-induced segmental instability ${ }^{3,10}$. In a biomechanical study of graded facetectomy, Abumi et al. ${ }^{1)}$, reported that medial facetectomy does not affect lumbarspinal instability whereas total facetectomy, even when created unilaterally, makes the lumbar spine unstable. In our study, we tried to avoid performing medial facetectomy to maintain spinal stability. To remove the ligamentumflavum, we simply cut the medial border of the facet using an inclined microscope. Preserving the facet joints during decompression could also produce less postoperative instability. Therefore, the risk of postoperative instability could be less common compared to conventional decompression with medial or total facetectomy. However, even minimal invasive bilateral laminotomy and simple ligamentectomy procedures can cause spinal instability.

\section{Disc herniation}

Multiple structural damage to the posterior element of the spine caused by the multilevel laminectomy and increased sagittal rotation may have stimulated nerve endings in and around the fibrous tissue of the discs and facet joints. Increased segmental motion of the vertebra stress on the motion segment by removal of posterior element may have caused not only low back pain but also disc herniation several years after decompressive laminectomy without fusion. However, in our study we experienced two cases of new lumbar disc herniation within 1 month after decompressive bilateral laminotomy. We do not know the exact mechanism underlying this type of complication, but we believe it is possiblethat direct damage to the annulus during decompression of the nerve roots by suction tip or micro-punch could cause disc herniation.

\section{Facet cyst}

Several hypotheses concerning the origin and development of juxtafacet cysts have been reported. These lesions have been described as originating from a synovium herniation through a defective joint capsule, from mucinous degeneration within the periarticular fibrous tissue in the ligamentum flavum, from repetitive microtrauma with focal hemorrhage, and from a part of the articular capsule of the facet joint or some distensible tissue penetrating into a ruptured degenerative ligamentum flavum ${ }^{2,4,8)}$. Ikuta et al. ${ }^{6}$ reported that the presence of segmental spinal instability, including degenerative spondylolisthesis and postoperative segmental spinal instability at the site of the cyst, are related to the development of postoperative intraspinal facet cysts. Furthermore, facet fluid detected on MR imaging was observed in all involved facet joints at the time postoperative intraspinal facet cyst development. After decompression surgery, a joint capsule of the facet joint can protrude into the spinal canal because the medial portion of the facet joint, from where the ligamentum flavum might have been removed during the decompression surgery, becomes weak. Evidence for the validity of this hypothesis can be derived from our study by the fact that postoperative intraspinal facet cysts developed within 12 months after surgery in all patients. In addition, mechanical stress, such as segmental spinal instability at the involved vertebral level, would aggravate the protrusion of the joint capsule. The mechanism underlying the spontaneous regression of intraspinal facet cysts is still unknown. It is thought that restoration of a destroyed facet joint by surgical invasion and restabilization of the involved spinal segment may cause the cysts to regress with histological changes ${ }^{6}$. In the presentstudy, spontaneous regression of the cysts with changes in the signal intensity on the radiological images was observed in all patients.

The design of this investigation has some limitations. First, the evaluation was performed in a non-randomized, retrospective fashion. Second, we examined the patients who had symptoms after surgery. If we included a more rigorous selection of asymptomatic patients for longer follow-up periods, more complications might have been observed. In spite of these 
limitations, we believe that the data obtained from a minimum follow-up of 24 months demonstrated several important results.

\section{CONCLUSION}

Decompressive bilateral laminotomy is believed to be effective for treating spinal stenosis and able to lessen the need for fusion. However, various symptomatic complications can still be observed after this procedure. It is important to be aware of the possibility of these various complications to improve the outcomes for surgical treatment of LSS.

\section{REFERENCES}

1. Abumi K, Panjabi MM, Kramer KM, Duranceau J, Oxland T, Crisco JJ: Biomechanical evaluation of lumbar spinal stability after graded facetectomies. Spine (Phila Pa 1976) 15: 1142-1147, 1990

2. Kao CC, Winkler SS, Turner JH: Synovial cyst of spinal facet. Case report. J Neurosurg 41:372-6, 1974

3. Kato Y, Panjabi MM, Nibu K: Biomechanical study of lumbar spinal stability after osteoplastic laminectomy. J Spinal Disord 11:146-50, 1998

4. Kjerulf TD, Terry DW Jr, Boubelik RJ: Lumbar synovial or ganglion cysts. Neurosurgery 19:415-20, 1986
5. Kim SW, Ju CI, Kim CG, Lee SM, Shin H: Minimally invasive lumbar spinal decompression: a comparative study between bilateral laminotomy and unilateral laminotomy for bilateral decompression. J Korean Neurosurg Soc 42:195-199, 2007

6. Ko Ikuta, Osamu Tono, Masayoshi Oga: Prevalence and clinical features of intraspinal facet cysts after decompression surgery for lumbar spinal stenosis. J Neurosurg Spine 10:617622, 2009

7. Park JY: Pathophysiology of lumbar spinal stenosis. Korean J Spine 1(4):407-416, 2004

8. Prestar FJ: Juxta facet cysts of the lumbar spine. Minim invasive. Neurosurg 39:45-49, 1996

9. Robertson PA, Grobler LJ, Novotny JE, Katz JN: Postoperative spondylolisthesis at L4-5. The role of facet joint morphology. Spine (Phila Pa 1976) 18:1483-90, 1993

10. Sharma M, Langrana NA, Rodriguez J: Role of ligaments and facets in lumbar spinal stability. Spine (Phila Pa 1976)20:887900, 1995

11. Sihvonen T, Herno A, Paljärvi L, Airaksinen O, Partanen J, Tapaninaho A: Local denervation atrophy of paraspinal muscles in postoperative failed back syndrome. Spine (Phila Pa 1976) 18:575-581, 1993

12. Thomé C, Zevgaridis D, Leheta O, Bäzner H, PöcklerSchöniger C, Wöhrle J, et al: Outcome after less-invasive decompression of lumbar spinal stenosis: a randomized comparison of unilateral laminotomy, bilateral laminotomy, and laminectomy. J Neurosurg Spine 3:129-41, 2005 